\title{
Using participatory design to determine the needs of informal caregivers for smart home user interfaces
}

\author{
Amy S. Hwang \\ Graduate Department of Rehabilitation \\ Science \\ University of Toronto \\ Toronto, Canada \\ amy.hwang@utoronto.ca
}

\author{
Khai N. Truong \\ Department of Computer Science \\ University of Toronto \\ Toronto, Canada \\ khai@cs.toronto.edu
}

\author{
Alex Mihailidis \\ Department of Occupational Science \\ and Occupational Therapy \\ University of Toronto \\ Toronto, Canada \\ alex.mihailidis@utoronto.ca
}

\begin{abstract}
Smart home technologies hold potential to help older adults with dementia complete activities independently, while alleviating the burden placed on their informal caregivers. Toward this goal, more research focus is needed to involve informal caregivers in the needs analysis and design of smart home user interfaces, for which they are likely to become primary users in the future. In this paper, we present our participatory design approach and the tensions that challenge user interface design for the intelligent $\mathrm{COACH}$ system that assists with activities of daily living in the home. Future work should involve older adults with dementia and other care stakeholders in user-centered design processes, and the development of systematic, interdisciplinary approaches for user interface design.
\end{abstract}

Keywords-intelligent systems; smart homes; dementia; informal caregivers; user interfaces; participatory design; caregiver burden

\section{INTRODUCTION}

The current dementia epidemic poses tremendous social and economic challenges to our health care and social systems. Most commonly affecting older adults [1], Alzheimer's disease (AD) related dementia is characterized by impairments in memory, thinking, behaviour and emotion [2], difficulty making appropriate judgments and responses [3], and trouble remembering sequences of steps in routine activities of daily living (ADLs) [4]. This loss of function may compromise an individual's independence in ADLs, thus, requiring some form of care. Older adults with dementia (OAWDs) usually want to continue living at home [5], but informal care (i.e., unpaid and typically from family members, close friends or neighbours) is uniquely demanding [6] and expensive [7]. Moreover, caregiver burden has been shown to have deleterious effects on caregivers' physical and mental health [6].

One strategy addressing these problems is the development of smart home technologies. To date, the field has predominantly focused on developing the "back end" of smart home systems; that is, validating engineering or computer science techniques that will enable systems to sense, learn, and appropriately react to what is occurring in the home environment. However, system efficacy alone cannot determine if technologies will meet the needs of real-world users, or guarantee if they are adopted and accepted in the home. Concurrent efforts must focus on designing the user interfaces (UIs) with which people will interact to operate and

Sponsored by the Alzheimer's Association's Everyday Technologies for Alzheimer's Care (ETAC) program and the Canadian Institutes for Health manage the systems. To this end, there is an immediate need to prioritize research efforts that will afford us a deeper understanding of different stakeholders' needs [8], and involve these stakeholders in the design of smart home UIs.

One important stakeholder group whose needs have not received considerable research focus is informal caregivers of OAWDs. Informal caregivers are well positioned to inform technology design for smart home UIs. As they are often the primary caregivers to OAWDs living at home [7], these individuals will likely be designated to purchase the technology, set it up in the home, and manage the information exchange with these systems. Although OAWDs are most often considered the primary users of "zero-effort" [9] smart home technologies, we argue that informal caregivers will become the primary users of smart home UIs. Thus, if smart home technologies hope to supplement care and alleviate caregiver burden [10], design that addresses the idiosyncratic needs of informal caregivers will be critical to future consumer satisfaction, and technology adoption and acceptance.

For this early exploration, we employed participatory design (PD) [11] to involve informal caregivers as co-designers of a UI prototype to manage the COACH system [13]. $\mathrm{COACH}$ is an intelligent system that leverages machine learning and computer vision to prompt OAWDs through ADLs. To date, $\mathrm{COACH}$ has demonstrated system efficacy during clinical trials supporting handwashing, and is currently undergoing further development to support additional ADLs. To improve its practicality for OAWDs living at home, this study targets informal caregivers as the primary users of this UI to manage COACH. Mutual learning [12] was facilitated, where participants offered their lived experiences in dementia caregiving, and we helped identify new opportunities for technology to support them. In this paper, we discuss our method and present our results as tensions that exist between different informal caregivers' needs. These tensions challenge our design and elucidate the concerns of this important stakeholder group related to introducing intelligent technologies systems in the home, and becoming the primary users of the UIs that administer, customize, and support these systems.

\section{RELATED WORK}

No high level empirical evidence has yet to demonstrate the impact of smart home technologies on quality of life, economic 
outcomes, costs to end consumers, or costs health care providers [15]. Moreover, only three intelligent assistive technologies have undergone clinical studies involving participants with dementia, while several others merely report case studies and prototype evaluations [8]. Relevant to our discussion, even fewer studies have investigated the "care networks" [16] of OAWDs in terms of how these stakeholders will use, support, and interact with smart home systems. Chan et al. [17] and Topo [18] both highlighted the major role caregivers will play in determining the conditions of smart home technologies, suggesting more focus on what will be needed to enable dementia caregivers to manage, support, and respond to these systems in the future.

\section{A. Examining caregivers needs for smart home technologies}

Rialle et al. [19] observed that caregivers' needs, perceptions, and expectations of assistive technologies supporting $\mathrm{AD}$ have been studied mainly using focus groups, interviews, questionnaires, and ethnographic studies. They administered a questionnaire to 270 family caregivers of AD to collect views on "care aiding" smart home technologies (e.g., fall detection systems). This study identified two main clusters of family caregivers: those who believed the technology could be helpful in supporting caregiving, and those who hostilely rejected the use of technology to aid care.

Rosenberg et al. [20] used a grounded theory approach to explore how "significant others" felt about using technology for their own use, and to support their relatives with dementia. Eight qualitative interviews $(n=8)$ and five focus group sessions $(n=11)$ were conducted to capture their experiences and ideas concerning technologies to support persons with dementia. This study revealed attitudes of readiness, both to support relatives with dementia in their technology use, and to become technology users themselves. Technologies that support ADLs should be integrated into habits, help persons with dementia retain and exercise their intact abilities, and be designed in form factors that avoid stigmatization.

Czarnuch and Mihailidis [21] surveyed 106 family caregivers using an online questionnaire to identify the needs of individuals with dementia for $\mathrm{ADL}$ completion, and to determine the features that would make assistive technologies most valuable. Factor analysis revealed that family caregivers found it most challenging to assist with ADLs that were more private in nature (e.g., in the bathroom), implying an opportunity for assistive technologies. Authors suggest family caregiver involvement in research studies should aim to educate these stakeholders on the potential of intelligent technologies to help overcome attitudinal challenges.

\section{B. User-centered design approaches involving dementia caregivers}

To understand different stakeholders' needs, Bharucha et al. [8] call for an urgent research focus on user-centered design in the development of smart home technologies. User-centered design invests substantial time and resources up front to gather users' needs, values, and preferences to ultimately optimize technology acceptance [22]. The design process is initiated by detailed analyses of users (e.g., physical, cognitive, demographic characteristics) and their social environments; and their tasks that technological innovations could potentially help them accomplish. This is followed by multiple iterations of design prototyping and user testing to drive development before real-world deployment.

Consolvo et al. [16] applied user-centered design to examine care coordination between different members of an elder's "care network" (e.g., family members, caregivers, healthcare professionals). Semi-structured interviews $(n=16)$ and follow-up diary studies $(n=3)$, drove the design of the CareNet display. This prototype was an ambient picture frame displaying an elder's photo and information about his or her status, activities, and whereabouts. CareNet was deployed as a technology probe for 3-week periods, and was updated by researchers who made ongoing calls to the elders and their care network members. Elders were found to have rich care networks with members of varying ages, relationships, and levels of involvement in their care, although communication is typically channeled through one designated caregiver. Resistance to care, care dynamics, and ongoing changes to care needs were noted as important design considerations.

The Keeping In Touch Everyday (KITE) project [23] aimed to create technology prototypes facilitating independence for people with dementia to get "out and about", while providing caregivers assurance of their safety. Individuals with dementia and their informal caregivers participated in a 3-stage participatory design process, involving focus groups $(n=21)$, participatory design workshops $(n=22)$, and the building $(n=3)$ of two prototypes: an armband and an electronic notepad. Accommodating illness progression and smaller, less stigmatizing form factors were key design recommendations.

The abovementioned work sets the stage for our study and highlights key research gaps which call for more focus on smart home UI design supporting OAWDs, and the consideration of informal caregivers as primary users of these interfaces. Our work uses the COACH system [13] as an example of a smart home technology in development, and employs participatory design to drive the initial design and formative evaluation of its first UI prototype.

\section{METHOD}

\section{A. Participants}

The first phase of our study was conducted with six participants who were all informal caregivers of older adults with dementia (OAWDs). Participants were recruited from a community-based non-profit charity providing outreach and respite care programs for caregivers of persons with AD. The inclusion criteria were fairly relaxed to explore a broad and diverse set of caregiving experiences, relationships with the OAWDs, preferences, attitudes, and perspectives. All recruited participants assisted with most or all of the following ADLs: bathing, toileting, handwashing, toothbrushing, dressing, meal preparation, and taking medications. Moreover, all participants had been providing informal care for at least six months to ensure they were well adjusted to and familiar with ADL support. Participants were qualified for the study based on at least 7 hours of caregiving per week, approximately half the average number of informal caregiving hours to older adults with mild dementia [24]. A description of participants is summarized in Table 1. 
TABLE I. DESCRIPTION OF PARTICIPANTS

\begin{tabular}{|c|c|c|c|c|c|}
\hline \multirow{2}{*}{$\begin{array}{c}\text { Participant } \\
\text { ID }\end{array}$} & Age & $\begin{array}{c}\text { Relationship to care } \\
\text { recipient }\end{array}$ & Living with care recipient & $\begin{array}{c}\text { Pegree of dementia } \\
\text { (self-reported) }\end{array}$ & $\begin{array}{c}\text { Self-reported \# ADLs requiring } \\
\text { assistance } \\
\text { (out of } 7 \text { ) }\end{array}$ \\
\cline { 2 - 6 } & Daughter & No, same apartment building & Mild-moderate & 6 \\
\hline J.L. & 55 & Daughter & No, within 5-min. drive & Moderate & 7 \\
\hline H.R. & 67 & Spouse & Yes & Mild-moderate & Moderate \\
\hline K.W. & 74 & Spouse & Yes & Moderate to severe & 7 \\
\hline T.J. & 77 & Daughter & $\begin{array}{c}\text { No, spends full days at nursing } \\
\text { home every other day }\end{array}$ & Severe & 7 \\
\hline M.V. & 37 & Daughter & Yes & 7 \\
\hline H.A. & 62 & &
\end{tabular}

\section{B. Phase 1: Group participatory design sessions}

We conducted two, 90-minute group participatory design sessions, one month apart. Sessions involved the six recruited participants, a professional faciliator (with a nursing background), the first author, and an additional member of the research team. Both sessions were preceded by 30 minutes of casual conversation over a light dinner to build rapport before the sessions commenced. Sessions were both video- and audio- recorded for later data analysis.

The first session aimed to familarize participants with the $\mathrm{COACH}$ system, discuss their needs and concerns related to introducing it in the home, and determine the design requirements for a UI. A brief animated video was displayed to depict $\mathrm{COACH}$ in a home scenario (Figure 1). The purpose of using an animated video was to illustrate the concept of $\mathrm{COACH}$ as a technology in development, and encourage feedback and criticism without the bias live action video may have imposed [25]. Discussion was guided to first elicit participants' initial reactions to and questions regarding $\mathrm{COACH}$, and then segued to what aspects of $\mathrm{COACH}$ participants would want to control, what information they would want the system to communicate, and when and how they would want to receive the desired information.

Participants were then asked to individually reflect on the discussion and write or sketch ideas, scenarios, or designs on their envisioned interaction with $\mathrm{COACH}$. Various examples were presented to facilitate this individual exercise, and creative writing and art supplies were provided. Each participant then presented her ideas to the group to generate questions, comments, and collaborative brainstorming. Before closing the session, all UI design requirements suggested during the session were summarized aloud for consensus. Researchers' field notes and participants' written material were collected for data analysis with video and audio recordings.

Audio recordings of the first session were professionally transcribed verbatim. The first author analyzed the transcript alongside salient excerpts of the video recordings, and organized the data into the following categories: a) issues raised by participants related to deploying $\mathrm{COACH}$ to the home; b) participants' ideas for improving $\mathrm{COACH}$; c) ADLs that participants felt $\mathrm{COACH}$ could assist with; d) features of $\mathrm{COACH}$ that participants would want to control; e) information participants would want to receive from $\mathrm{COACH}$; f) preferred technological devices and modalities for interaction with $\mathrm{COACH} ; \mathrm{g}$ ) individual participants' ideas and designs. A session summary was developed and sent to all participants (via mail or email) two weeks before the second session to reiterate the ideas discussed. Based, on categories c), d), e), and f), the first author created preliminary UI designs (using Axure RP software) in preparation for the second group session.

The second session aimed to gather participants' feedback and design recommendations on preliminary UI designs. The first author reiterated the key research questions of the study to help refocus participants on designing a UI for their own interaction with $\mathrm{COACH}$. Participants were then divided into two groups of three. The first group, facilitated by the facilitator and first author, discussed the UI designs for the "setup wizard" and home screen. The second group, facilitated by a student research assistant, discussed the UI designs for modifying existing activities and adding custom activities in $\mathrm{COACH}$. Both groups were provided with writing and art supplies to actively participate in this exercise. Upon reconvening as one large group, the key design recommendations and additional functional requirements were summarized aloud for further group discussion. Research team field notes and participants' written material were collected for analysis with video and audio recordings.

Data from the second group session were analyzed by a method similar to the first session. Audio recordings were professionally transcribed verbatim, reviewed with support from video recordings, and analyzed through discussion between all authors. A list of suggested UI revisions and

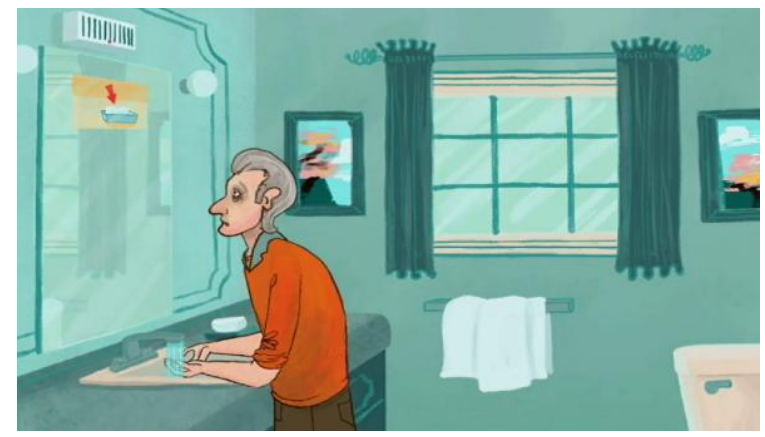

Figure 1. An animated video depicting the $\mathrm{COACH}$ system in a home environment, assisting an OAWD during handwashing. 
additional functional requirements was generated. At this time, all authors agreed it would be valuable to conduct task-based testing of a paper prototype of the next design iteration before proceeding to functional prototype development.

\section{Phase 2: Usability testing with a paper prototype}

The second UI design iteration was developed using Balsamiq Mock-Ups software and built into a paper prototype for usability testing on five tasks: a) initial COACH set-up in the home, b) modifying the steps, prompts and triggers of a pre-programmed activity (i.e., tooth-brushing), c) obtaining a report from $\mathrm{COACH}$ on tooth-brushing over a specified period of time (see Figure 2), d) creating and adding a custom activity (i.e., making a cup of tea) for $\mathrm{COACH}$ to support, and e) using video and audio capabilities to remotely monitor and communicate with an OAWD while away from the home.

Two participants from Phase 1, M.V. and H.A., agreed to participate in individual usability test sessions in their homes. Participants were selected by the research team based on their demonstrated engagement, understanding of the key research questions, effective communication of feedback, and willingness and availability participate.

At each individual session, the second author played the role of the "facilitator", providing instructions throughout the session, introducing each task, and guiding the discussion with the participant. The first author, the prototype designer, played the role of the "computer", switching the pages of the prototype during tasks. Where needed, the first author also provided clarification to the participant and asked questions to clarify her feedback and design recommendations. Both authors took field notes throughout the session.

To introduce each task, participants were given one-page task descriptions containing the scenario, tasks to complete using the paper prototype, and any additional information required to complete the task. Participants were asked to read the descriptions aloud before starting each task. While completing the tasks, participants were encouraged to think aloud (i.e., provide running commentary on what they are thinking and doing) [26], critique the content and layout of the interface, and ask questions if they experienced difficulty. Although fictitious scenarios and characters were used to constrain user actions on the low-fidelity prototype, participants were strongly encouraged to reflect and comment on the appropriateness of the design to their unique situations. When participants experienced difficulties completing the tasks, the second author asked them to explain the usability problems and avoided guiding or correcting erroneous actions. Participants were also invited to write or draw their suggested revisions directly on the paper prototype. At the end of each session, both authors engaged in an open discussion with participants on their overall reactions to and feedback on the prototype design, and the $\mathrm{COACH}$ system in general. All paper prototype mark-ups, field notes, and video and audio recordings were collected for data analysis.

Following the usability test sessions, the first author reviewed all data and generated a list of suggested prototype revisions, user recommendations, and design considerations.

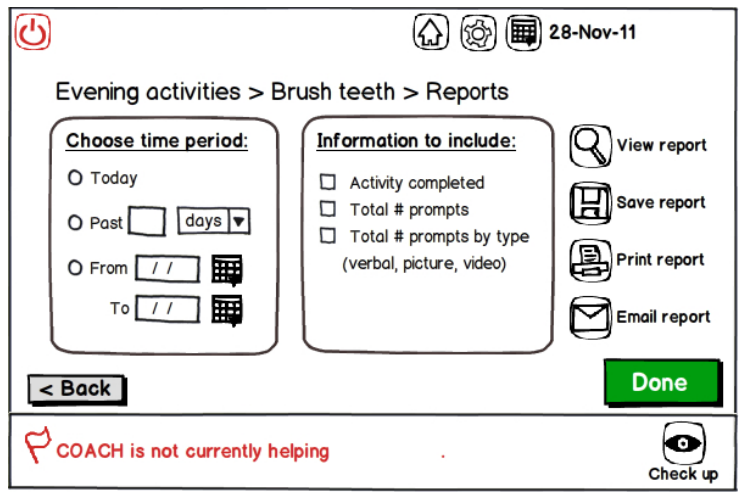

Figure 2. A sample of our paper prototype used for the task of obtaining a report from $\mathrm{COACH}$ (Phase 2).

Ongoing discussion between all authors is currently underway in preparation for functional prototype development.

\section{RESULTS}

During both group and individual sessions, participants expressed general enthusiasm toward exploring how $\mathrm{COACH}$ could help them in their caregiving roles. Several conflicting needs, however, were identified which posed challenges to our UI design. We present these as design tensions which exemplify domain issues in need of consideration for future smart home UI design work.

\section{A. Customizing COACH vs. shifting caregiver burden}

Participants believe $\mathrm{COACH}$ will only be useful to them if they can customize the system to their unique care situations and preferences. Participants wanted to be able to select among activities already pre-programmed into the system, and create new custom activities with which $\mathrm{COACH}$ could assist: "I'd like the preprogrammed [activities that are] common as people age or as people deteriorate, [and the] ability to add specifics...something that's not on your list." The ability to customize or modify the steps within an activity was also important, although participants predicted that doing so may be onerous and time-consuming: "So you're going to have to basically pay very close attention initially to that person's daily routine, and try to break down how long it takes you to prompt that person to do something, and average it up..." Moreover, participants thought it was crucial to be able to personalize the prompts that $\mathrm{COACH}$ would deliver, to ensure they were familiar, appropriate, and sensitive to the OAWD: "I see how my husband reacts... if anybody would say 'great job', he would be kind of put down... at the early stage, you really have to worry an awful lot about their pride... and consider their feelings, you can't take over." Participants also wanted to be able to determine the criteria for activating specific prompts (e.g., deliver a verbal prompt when the OAWD has not moved on to the next step after 45 seconds).

Additional requirements included the ability to define how and when $\mathrm{COACH}$ would alert caregivers for assistance; generate ad hoc reports based on defined time periods and information criteria; create profiles to enable multiple caregivers to interact with $\mathrm{COACH}$; choose preferred technological devices (e.g., PC, television, mobile phone) and modalities (e.g., email, text message, voice alert) to control 
and receive information from $\mathrm{COACH}$; and define the language $\mathrm{COACH}$ uses to deliver prompts.

In conflict with these numerous functional requirements, however, was the unanimous concern that introducing $\mathrm{COACH}$ in the home would shift caregivers' burden from caregiving to managing the technology: "And also you want $\mathrm{COACH}$ to be helping you, not giving you something extra that you've got to do." Most felt they did not have time to program, administer, and maintain the system, and would not replace spending time with and caring for their loved ones to "deal with" the technology. Despite engaging and optimistic discussion around this conflict, participants could not suggest any resolutions.

\section{B. Supporting vs. replacing human caregiving}

Participants were receptive and enthusiastic to the idea of leveraging modern technology to alleviate their burden, and promote independence for OAWDs, but emphasized that no system could replace human caregiving: "Well...the technology is incredible and it's amazing in terms of its growth, but it's still technology, and as good and as sensitive and as incredulous as technology is, it's not going to replace another human being. So we need to keep that in perspective in terms of what are we expecting from a machine." It was clear they valued and took pride in their caregiving roles, but saw opportunities that technology could help alleviate workload if designed to be sensitive to and compatible with their needs.

A few different opportunities for $\mathrm{COACH}$ to supplement human caregiving were suggested. One participant, who struggled with constantly repeating herself in caring for her husband, felt $\mathrm{COACH}$ may be able to assist in this area: "It's alleviating...it's alleviating you repeating yourself..." Another participant, who viewed herself as a "manager" of several personal support workers who provided care for her mother, suggested $\mathrm{COACH}$ could potentially help her coordinate care: "I would like COACH to be an all encompassing item because I have different [personal support workers] coming in [at] different times." Participants also implied that the degree of potential danger may differentiate between the caregiving tasks they would allow $\mathrm{COACH}$ to support, and those tasks they would only entrust to themselves or personal support workers: "The technology would have to control things that are not safety issues. I would not feel comfortable with technology controlling something that could be potentially...a serious danger." Notwithstanding these positive suggestions, however, apprehension seemed to remain among participants: "So you're going to have your whole house literally wired for sound and sight...that won't take the place of [a personal support worker] because once you have that information somebody has to act on it...[because] a technology isn't going to do whatever that is. " These attitudes are to be expected and could be attributed to fear for the safety of their loved ones, lack of trust, and lack of knowledge on the capabilities of intelligent systems.

\section{Safety vs. privacy}

Safeguarding against harm was arguably the greatest concern expressed by participants, despite all considerations related to respect for one's privacy and autonomy. In terms of UI requirements, participants desired real-time system status updates (i.e., displayed as text across the bottom margin of the home screen); video-monitoring of different areas of the home, with options to view either in real-time or at a later time; and a two-way channel (e.g., "intercom") enabling ondemand voice communication with the OAWD. "There needs to be almost a constant link so that you will know in the moment when something isn't going right." Moreover, although it was considered beyond the scope of this study, participants expressed the desire to track (via GPS) and communicate with OAWDs who leave home to mitigate the risks of wandering. Although tracking and video surveillance were acknowledged to compromise the privacy of OAWDs, from the perspective of these informal caregivers, safety was considered paramount and should take the highest priority: "It's like policing. It's like invading a person's privacy...but for their own sake." Moreover, the ability to check in with and monitor their loved ones would provide more reassurance when caregivers needed to leave the house, which was identified as a key concern: "Leaving the house was important in stage one for me."

\section{Decoupling features vs. form factor}

Participants had diverse preferences for devices and modalities for their interaction with $\mathrm{COACH}$. Some participants wanted to use familiar devices they had experience using (e.g., televisions, home telephones, answering machines). Those participants who used desktop or laptop PCs liked the option of receiving non-urgent information by email. The regular mobile phone or smart phone users also liked the option of receiving alerts via voice call, text message, or email: "I think a phone would be best because it's mobile. You're not always at your computer or at your TV [at home]. And you're always carrying your cell phone." When we presented different touch-screen tablet devices (i.e., iPad, BlackBerry Playbook, Samsung Galaxy Tab), most were agreeable to their form factors, although preferences were divided on carrying the device outside the home. After usability test sessions examining the paper prototype, participants also suggested a fixed-location intercom interface in the home and a wrist-wearable alert device that could be transferred between multiple caregivers (e.g., family members, personal support workers). These mixed preferences, which seemed to vary between individuals, related more to form factor and less to UI features. This suggests a need to decouple these requirements and develop functionality that will work across a variety of form factors, allowing users to select which features match their preferences for particular devices.

\section{E. Timely delivery of information vs. disruptions}

Participants felt it was important to receive a variety of information from $\mathrm{COACH}$. Again, prioritizing the safety of their loved ones, they wanted the system to call if their assistance was needed, or to alert them of an emergency situation: "There also needs to be at that point, if that person isn't redirected to do the appropriate behaviour at that time...some sort of alert to that other person so they can come in and actively take over from $\mathrm{COACH}$, to get that thing done. 
Once they're redirected again, maybe I could leave." Furthermore, participants felt that reports from $\mathrm{COACH}(e . g$., showing patterns of activity completion, responsiveness to prompts, specific problem steps) would be helpful in tracking health status over time, and suggest when medical visits should be initiated: "I'm not saying [there should be] printout on a regular basis, [just] as required...because sometimes my mother has a bad evening [and the] next day she's fine...but then if that runs several days in a row, you've got to know when it's time to talk to the doctor." On the other hand, participants consistently expressed concerns about being disrupted or bothered by the system, particularly given their current workloads and demands. Successful design would need timely delivery of information in a non-disruptive and unobtrusive manner. Moreover, in reporting information to informal caregivers, what is considered "acceptable" or "normal" activity completion is highly individualized, subjective, and dependent on the specific activity.

\section{F. Defining and scheduling tasks vs. flexibly accommodating for abilities and home life}

Arguably, our most interesting finding was the desire to define and schedule activities in advance, yet be able to make modifications quickly and easily when abilities, preferences, or circumstances change: "So if I were doing it, I wouldn't like to program it on a daily basis. I would like to sit it down for a month at least and program the monthly activities...but there also has to be a way for me of course to come in [because] things change...so I have to be able to come in and change things very simply." First, participants called for a system that could flexibly accommodate ongoing fluctuations in mood and functional ability, as well as the progressive decline of abilities due to disease progression: "I think it would be necessary [to customize] because each person is an individual... and the needs and abilities are different...it would have to be able to customize in terms of disease progression... and what works today may not work tomorrow. So, there would have to be some sort of flexibility built into it."

Moreover, it became clear that the need to define and schedule activities would be challenged by the dynamic, improvisational nature of routines and activities in the home. For example, certain activities that caregivers consider necessary to complete may not be bound to specific start times: “... because I don't know about you, but I get up sometimes at 7 o'clock, sometimes at 6 o'clock...so that flexibility has to also be built into it." Groups of activities, may need to take place in specific orders (e.g., a meal must precede taking medication), but may or may not be flexible with respect to time or time of day. Then, other activities may be entirely optional with respect to time, mood, and preference, where it may be valuable for $\mathrm{COACH}$ to suggest and then support OAWDs during times of leisure or inactivity. Discussions with participants related to the nature and parameters of activities proved to highlight, what we would argue to be, our greatest design challenge encountered in this study: "How do you program the unpredictable? How do you program something that's not routine? How do you program into the technology the specific personality of how the individual is?"

\section{DISCUSSION}

Our study has contributed a novel description of needs from informal caregivers, who are positioned to be the primary users of future smart home UIs. To date, the perspectives, preferences, challenges, and requirements of informal caregivers have been underrepresented in the smart home literature. By instantiating our exploration using the $\mathrm{COACH}$ system, and employing participatory design and technology probes, participants were able to conceptualize the capabilities of a future home technology, articulate predicted challenges, and recommend design improvements. Our study has enabled us to be conversant in more than the UI design requirements for $\mathrm{COACH}$; it has elucidated the tensions and social considerations that may facilitate, or impose barriers to, the design of smart home technologies supporting OAWDs and informal dementia care.

On one hand, studies to date suggest that some informal caregivers embrace the development of smart home technologies (e.g., [14][19]) and seem receptive to these technologies in a hypothetical sense [8]. Other studies, however, have revealed resistant and rejective attitudes toward home technologies supporting dementia care. Czarnuch and Mihailidis [21] found that caregivers did not feel they would have time to interact with assistive technology and, thus, were not interested to learn how to use them. Rialle et al. [19] also found a well-defined cluster of family caregivers who rejected the use of smart home technology with hostility. In our study, we were confronted with similarly varying attitudes toward intelligent technologies supporting dementia care. Initially, during group sessions, participants had difficulty conceptualizing the capabilities of $\mathrm{COACH}$ and focusing on how they, as informal caregivers, would want to use and interact with the system. Through further group discussion and individual usability sessions, however, they were able to better appreciate our design objectives and expressed increased enthusiasm toward the technology. To Czarnuch and Mihailidis' [21] suggestion that research studies should help educate caregivers on technological capabilities, we propose our participatory methodology as an example of a successful design partnership, which informed caregivers on the potential of intelligent technology, helped them acknowledge and overcome deferential attitudes, and empowered them as codesigners of future solutions.

The tension between high customizability and potential shift of burden is also central to our design challenge. Coughlin et al. [14], citing Engstrom et al. [27], emphasized the importance of considering the perceived "hassle factor" of smart home technologies, where the "related logistics associated with installation, correct use, maintenance, etc., may be just great enough to outweigh the possible benefits of inviting new technology into an older person's daily routine." Our study confirmed this perception, as participants questioned the usefulness of $\mathrm{COACH}$ if its presence would simply shift their burden from providing care to programming and administering the system. Thus, as articulated by Davidoff et al. [28], it is not sufficient to examine system usability alone; more research focus on the utility of smart home systems will be critical to future technology adoption and acceptance. 
As described in the tension between features and form factor, caregivers expressed different preferences for devices and modalities for their interaction with $\mathrm{COACH}$. Surprisingly, most participants were partial to the tablet computers presented (e.g., iPad, BlackBerry Playbook) in terms of form factor and touch-screen interaction, despite having no previous experience of use. This varies from Consolvo et al.'s [16] recommendation that existing technologies (e.g., telephones, televisions) should be exploited to augment existing habits, but confirms their finding that caregivers desire technologies with mobile form factors. Without actual home deployments to explore routines and habits in relation to these technologies, however, it may be premature to determine caregivers' modality preferences. We argue that features and form factor need to be decoupled to identify the desired functionality before assigning specific features to a mix of preferred devices. Form factor should be seen as user-specific and driven by preference, experience, and willingness to learn proficiencies on new technologies. Our work recommends that designers strive toward functionality that will work across a variety of form factors, where users can select features that match their device and modality preferences.

The tension between defining and scheduling activities, and allowing enough flexibility to accommodate an OAWD and his or her dynamic home life, further emphasizes the need for revised approaches to smart home UI design. Related studies (e.g., [18][23]) have confirmed our findings that technologies need to accommodate for both the fluctuating and degenerative nature of dementia. Although outside the context of supporting OAWDs and dementia care, we consider Davidoff et al.'s [28] work exploring smart home systems and their potential to improve families' sense of control in everyday life. This work argues that an end-user programming approach conflicts with the organic, dynamic, and improvisational nature of home environments. Families' activities and routines are not easily mapped to well-defined programming tasks. Rather, families develop rhythms of routines and plans that evolve organically but are also subject to breakdown with cascade effects. As such, systems should be designed to support planned activities, but must have enough flexibility to adapt, recover from, and respond to change when confronted with exceptions or breakdowns. As the burden of informal dementia care seems to carry similar undertones (i.e., competing life demands, loss of control, stress), we identify nuances that build on this related work. We have learned that the routines and ADLs of an OAWD vary on several parameters, including necessity, frequency and duration; time or time of day; dependencies on or relationships to other activities; and his or her abilities, moods, and preferences. Replacing end-user programming approaches with more descriptive methods, such as ethnographic and qualitative research, may help us better understand this phenomenon and design systems more likely to be embraced and accepted.

\section{CONCLUSION \& FUTURE WORK}

Our work hopes to pave the way for a greater focus on innovative approaches to the design of smart home UIs, to support informal dementia care, and improve independence for OAWDs. Moreover, we hope to motivate more research on designing to meet the needs of informal caregivers, not only as "proxies" representing the needs of individuals with disability, but as a primary user group for the UIs that may facilitate acceptability or adoption of intelligent systems. The design tensions presented aim to stimulate conversation and encourage creative collaboration across human-computer interaction, pervasive and ubiquitous computing, clinical and biomedical engineering, health and rehabilitation science, design, and the relevant social sciences.

Although this study purposefully targeted the needs, preferences, and opinions of informal caregivers as the primary users of smart home UIs, we feel that involving OAWDs in future design may help to clarify and potentially resolve tensions that exist between user groups. "What is right for the elder from a health perspective is not necessarily right for the elder emotionally [16]." Several recent studies (e.g., [16][23][29][31][32]) have demonstrated the feasibility and value of involving OAWD in the design process. Involving these care recipients will help us better describe our findings related to issues of pride and privacy that seem to be in conflict with caregivers' priorities of ensuring safety for OAWDs, which is well documented in the literature (e.g., [19][20][30][32]). Moreover, this will create opportunities to elicit the idiosyncratic needs of OAWDs, empower them through participation, deepen our knowledge of care dynamics, and address identified tensions through thoughtful design collaboration.

Our study also calls for further investigation of the diversity of informal caregivers and, more holistically, networks of dementia care, which extend to formal caregivers and professionals. Our findings were limited by a small sample of female informal caregivers, most of whom are older adults themselves. Although the common profile of a dementia caregiver is a female spouse or child, Brodaty and Donkin [7], citing 2008 statistics from the Alzheimer's Association, report that $40 \%$ of family caregivers of older adults are now male, an increase from $21 \%$ in 1996 . Moreover, as seen in our study and reported in the literature, caregiving burden differs between spouses and adult children. Spouses are likelier to live with the older adults, provide more direct care, and experience more stress and emotional loss, whereas adult children and children-in-law tend to arrange for others to provide care (e.g., personal support workers) due to a greater number of conflicting personal responsibilities (e.g., career, young children or adolescents) [7][33]. Consolvo et al. [16] also suggest the need to understand care networks, arguing that overlooking issues of caring as a whole will limit the impact of technologies in real-world deployments. Although care is typically channelled through and coordinated by one primary caregiver [16], all of our participants relied to some extent on formal care (e.g., personal support workers, nursing home staff), implying that the utility of smart home UIs may be improved if expanded to support care coordination. To further examine social dynamics, future work should also explore the sociocultural factors that influence user needs [21], as well as the potential impact of intelligent technologies on the dynamics and relationships within care networks, and between professionals, caregivers, and OAWDs.

To resolve the articulated design tensions and address the complex issues described, we close by advocating for the 
development of rigorous, systematic processes to guide smart home UI design. In our study, we drew upon participatory design and the use of technology probes (i.e., paper prototypes) to elicit needs and drive UI prototype development. As Davidoff et al. [28] suggested, however, methods employed to study typical users seem inadequate for studying needs, routines, and activities of individuals in their home environments. Thus, creative collaborations and novel methodological approaches are needed to empower dementia caregivers and OAWDs as co-designers, and promote ultimate adoption and acceptance in the future.

\section{ACKNOWLEDGMENT}

The authors wish to thank Stephen Czarnuch, Jennifer Boger, Frank Li, Sen Hirano, Rita Vanderlaan, Dr. Jill Cameron, and the staff at Circle of Care for their support and contributions to this research.

\section{REFERENCES}

[1] A. Wimo and M. Prince, "World Alzheimer's Report 2010: The Global Economic Impact of Dementia," Alz. Dis. Intl., London, UK, Sept. 2010.

[2] Alzheimer's Disease International. "The Global Voice on Dementia." Internet: http://www.alz.co.uk/about-dementia, [Feb. 15, 2012].

[3] J. Diamond, "A Report on Alzheimer's Disease and Current Research," Alz. Soc. Can., Toronto, ON, 2008.

[4] A. Mihailidis, J. C. Barbenel, and G. Fernie, "The Efficacy of an Intelligent Cognitive Orthosis to Facilitate Handwashing by Persons with Moderate to Severe Dementia," Neuropsychological Rehabilitation, vol. 14, pp. 135-171, 2004.

[5] J. Van Hoof and H. Kort, "Supportive Living Environments: a First Concept of a Dwelling Designed for Older Adults with Dementia," Dementia, vol. 8, pp. 293-316, 2009.

[6] R. Schulz and L. M. Martire, "Family Caregiving of Persons with Dementia: Prevalence, Health Effects, and Support Strategies," American Journal of Geriatric Psychiatry, vol. 12, pp. 240-249, 2004.

[7] H. Brodaty and M. Donkin, "Family Caregivers of People with Dementia," Dialogues in Clinical Neuroscience, vol. 11, pp. 217-228, 2009.

[8] A. J. Bharucha, V. Anand, J. Forlizzi, M. A. Dew, C. F. Reynolds, S. Stevens (3rd), and H. Wactlar, "Intelligent Assistive Technology Applications to Dementia Care: Current Capabilities, Limitations, and Future Challenges," American Journal of Geriatric Psychiatry, vol. 17, pp. 88-104, 2009.

[9] A. Mihailidis, J. Boger, J. Hoey, and T. Jiancaro, "Zero Effort Technologies: Considerations, Challenges and, Use in Health, Wellness, and Rehabilitation," in Synthesis lectures on assistive, rehabilitative, and health-preserving technologies, R. M. Baecker, Ed., San Rafael, CA: Morgan \& Claypool Publishers, 2011.

[10] J. Boger and A. Mihailidis, "The Future of Intelligent Assistive Technologies for Cognition: Devices Under Development to Support Independent Living and Aging-with-Choice," NeuroRehabilitation, vol. 28, pp. 271-280, 2011.

[11] M. Skeels and W. Pratt, "Participatory Design with Health Consumers," in AMIA Annual Symposium, Washington, DC, 2008, p. 1136.

[12] M. Kyng, "Designing for Cooperation: Cooperating in Design," Communications of the ACM, vol. 34, pp. 65-73, 1991.

[13] Mihailidis, J. N. Boger, T. Craig, and J. Hoey, "The COACH Prompting System to Assist Older Adults with Dementia Through Handwashing: an Efficacy Study," BMC Geriatrics, vol. 8, 2008.

[14] J. F. Coughlin, L. A. D'Ambrosio, B. Reimer, and M. R. Pratt, "Older Adult Perceptions of Smart Home Technologies: Implications for Research, Policy and Market Innovations in Healthcare," Proc. IEEE Eng. Med. Biol. Ann. Conf., Lyon, France, 2007.
[15] S. Martin, G. Kelly, G. Kernohan, B. McCreight, and C. Nugent, "Smart Home Technologies for Health and Social Care Support," Cochrane Database of Systematic Reviews, vol. 2008, 2009.

[16] S. Consolvo, P. Roessler, B. E. Shelton, A. LaMarca, and B. Schilit, "Technology for Care Networks of Elders," Pervasive Computing, vol. 3, pp. 22-29, April-June 2004.

[17] M. Chan, D. Esteve, C. Escriba, and E. Campo, "A Review of Smart Homes: Present State and Future Challenges," Computer Methods and Programs in Biomedicine, vol. 91, pp. 55-81, 2008.

[18] P. Topo, "Technology Studies to Meet the Needs of People with Dementia and Their Caregivers: a Literature Review " Journal of Applied Gerontology, vol. 28, pp. 5-37, 2009.

[19] V. Rialle, C. Ollivet, C. Guigui, and C. Hervé, "What Do Family Caregivers of Alzheimer's Disease Patients Desire in Smart Home Technologies? Contrasted results of a wide survey," Methods of Information in Medicine, vol. 47, pp. 63-69, 2008.

[20] L. Rosenberg, A. Kottorp, and L. Nygård, "Readiness for Technology Use with People with Dementia: the Perspectives of Significant Others," Journal of Applied Gerontology, 2011.

[21] S. Czarnuch and A. Mihailidis, "The Design of Intelligent In-Home Assistive Technologies: Assessing the Needs of Older Adults with Dementia and Their Caregivers " Gerontechnology, vol. 10, pp. 169-182, 2011.

[22] K. Vredenburg, S. Isensee, and C. Righi, User-Centered Design: an Integrated Approach. Upper Saddle River, NJ: Prentice Hall PTR, 2002.

[23] L. Robinson, K. Brittain, S. Lindsay, D. Jackson, and P. Olivier, "Keeping In Touch Everyday (KITE) Project: Developing Assistive Technologies with People with Dementia and Their Carers to Promote Independence," International Psychogeriatrics, vol. 21, pp. 494-502, June 2009.

[24] K. M. Langa et al., "National Estimates of the Quantity and Cost of Informal Caregiving for the Elderly with Dementia," Journal of General Internal Medicine, vol. 16, pp. 770-778, 2001.

[25] K. M. Sellen, M. Massimi, D. M. Lottridge, K. N. Truong, and S. A. Bittle, "The People-Prototype Problem: Understanding the Interaction Between Prototype Format and User Group.," in CHI '09, Boston, MA, 2009, pp. 635-638.

[26] J. Nielsen and J. Levy, "Measuring Usability: Preference vs. Performance," Communications of the ACM, vol. 37, pp. 66-75, 1994.

[27] M. Engstrom, R. Lindqvist, B. Liunggren, and M. Carlsson, "Relatives' Opinions of IT Support, Perceptions of Irritations and Life Satisfaction in Dementia Care," Journal of Telemedicine and Telecare, vol. 12, pp. 246-250, 2006.

[28] S. Davidoff, M. K. Lee, C. Yiu, J. Zimmerman, and A. K. Dey, "Principles of Smart Home Control," in Ubicomp 2006: Lecture notes in computer science, P . Dourish and A. Friday, Eds., Springer Berlin / Heidelberg, 2006.

[29] G. E. Lancioni et al., "Technology-Aided Pictorial Cues to Support the Performance of Daily Activities by Persons with Moderate Alzheimer's Disease," Research in Developmental Disabilities, vol. 33, pp. 265-273, 2012.

[30] R. Landau, G. K. Auslander, S. Werner, N. Shoval, and J. Heinik, "Families' and Professional Caregivers' Views of Using Advanced Technology to Track People with Dementia," Qualitative Health Research, vol. 20, pp. 409-419, 2010.

[31] R. Orpwood et al., "Designing Technology to Improve Quality of Life for People with Dementia: User-Led Approaches," Universal Access in the Information Society, vol. 9, pp. 249-259, 2010.

[32] A. Olsson, M. Engström, K. Skovdahl, and C. Lampic, "My, Your and Our Needs for Safety and Security: Relatives' Reflections on Using Information and Communication Technology in Dementia Care," Scandinavian Journal of Caring Sciences, pp. 104-112, 2011.

[33] M. Pinquart and S. Sörensen, "Spouses, Adult Children, and Childrenin-Law as Caregivers of Older Adults: a Meta-Analytic Comparison," Psychology and Aging, vol. 26, pp. 1-14, 2011. 